\title{
When and how do children develop knowledge of verb argument structure? Evidence from verb bias effects in a structural priming task
}

\author{
Michelle Peter ${ }^{\mathrm{a}, *}$, Franklin Chang ${ }^{\mathrm{a}}$, Julian M. Pine ${ }^{\mathrm{a}}$, Ryan Blything ${ }^{\mathrm{b}}$, Caroline F. Rowland ${ }^{\mathrm{a}}$ \\ ${ }^{a}$ Department of Psychological Sciences, Institute of Psychology, Health \& Society, University of Liverpool, Eleanor Rathbone Building, Liverpool L69 7ZA, UK \\ ${ }^{\mathrm{b}}$ Max Plank Child Study Centre, School of Psychological Sciences, University of Manchester, Manchester M13 9PL, UK
}

\section{A R T I C L E I N F O}

\section{Article history:}

Received 18 January 2014

revision received 4 December 2014

Available online 20 January 2015

\section{Keywords:}

Structural priming

Verb bias

Lexical boost

Prime surprisal

Error-based learning

Associative learning

\begin{abstract}
A B S T R A C T
In this study, we investigated when children develop adult-like verb-structure links, and examined two mechanisms, associative and error-based learning, that might explain how these verb-structure links are learned. Using structural priming, we tested children's and adults' ability to use verb-structure links in production in three ways; by manipulating: (1) verb overlap between prime and target, (2) target verb bias, and (3) prime verb bias. Children (aged 3-4 and 5-6 years old) and adults heard and produced double object dative (DOD) and prepositional object dative (PD) primes with DOD- and PD-biased verbs. Although all age groups showed significant evidence of structural priming, only adults showed increased priming when there was verb overlap between prime and target sentences (the lexical boost). The effect of target verb bias also grew with development. Critically, however, the effect of prime verb bias on the size of the priming effect (prime surprisal) was larger in children than in adults, suggesting that verb-structure links are present at the earliest age tested. Taken as a whole, the results suggest that children begin to acquire knowledge about verb-argument structure preferences early in acquisition, but that the ability to use adult-like verb bias in production gradually improves over development. We also argue that this pattern of results is best explained by a learning model that uses an error-based learning mechanism.
\end{abstract}

(c) 2014 Elsevier Inc. All rights reserved.

\section{Introduction}

A critical part of language acquisition is learning verb argument structure. Verb argument structure refers to the fact that in language, verbs can only occur in, or prefer to occur in, particular syntactic structures; and these verbstructure links need to be learnt if children are to produce well-formed sentences (for example, verbs like "sold" can occur in dative structures (the rock star sold the undercover agent some cocaine), but not in intransitive structures (*the

\footnotetext{
* Corresponding author.

E-mail address: Michelle.Peter@liv.ac.uk (M. Peter).
}

rock star sold)). Although argument structure is one of the most important components of syntactic development, we know very little about when and how children learn to link verbs to particular syntactic structures. In this work, we investigated the effect of verb bias on structural priming to determine when children learn verb-structure links, and to examine the nature of the learning mechanism behind the development of these links.

Structural priming has traditionally been used as a way to investigate the nature of adult syntactic representations, but it can also be used to investigate the way in which these representations interact with the verb lexicon. A number of priming studies have shown that adult speakers 
tend to repeat the syntactic structure of the sentences that they have recently encountered. For example, in a wellknown study by Bock (1986), adults were more likely to describe a target picture using a prepositional object dative (e.g., The man is reading a story to the boy) if they had just produced a prime sentence that was also a prepositional object dative (e.g., The rock star sold some cocaine to an undercover agent). This structural priming effect is not contingent on non-syntactic factors (such as the prosody of sentences or the thematic roles played by arguments), nor does it rely on the repetition of words across sentences. Thus, the phenomenon of structural priming is widely interpreted as evidence that adults have abstract representations of syntax that are stored independently of lexical items (e.g., Bock, 1989; Cleland \& Pickering, 2006; Noppeney \& Price, 2004).

The fact that structural priming does not rely on superficial comparisons between lexical items is now wellestablished. Nevertheless, it has been found that adults' knowledge of a verb's preferred argument structure (verb bias) influences their structure choice during priming tasks. Priming effects tend to be larger when prime and target sentences share a verb (the lexical boost; Pickering \& Branigan, 1998), and the syntactic preference of both the target verb (target verb bias; Gries, 2005) and the prime verb (Bernolet \& Hartsuiker, 2010; Jaeger \& Snider, 2013) affect the size of the structural priming effect. For example, both Bernolet and Hartsuiker, and Jaeger and Snider have shown that priming is stronger when the prime verb's bias does not match the prime structure in which it is presented - a phenomenon called prime surprisal. Taken together, these findings demonstrate that, although adults have abstract representations of syntactic structure, they also store links between verbs and the structures in which these verbs occur, and that these links can influence structural priming.

Structural priming has also been used in the language acquisition literature to investigate the nature of children's early syntactic representations (e.g., Bencini \& Valian, 2008; Messenger, Branigan, \& McLean, 2011; Shimpi, Gámez, Huttenlocher, \& Vasilyeva, 2007; Thothathiri \& Snedeker, 2008a). Developmental studies have established that children have acquired at least some abstract representations that enable them to generalise across similarly-structured sentences by around the age of three years (though see Savage, Lieven, Theakston, \& Tomasello, 2003, for contradictory evidence). However, an important question not addressed by these studies is how children's knowledge of syntactic structure interacts with their knowledge about the behaviour of particular verbs (verb argument structure). Thus, unlike the adult priming literature, the child priming literature has little to say about when children develop links between abstract syntactic representations and their developing verb lexicon, and even less about the mechanisms that might mediate this relationship.

One exception to this pattern is a recent study by Rowland, Chang, Ambridge, Pine, and Lieven (2012). Using a structural priming task, Rowland et al. found evidence of abstract priming in both children (aged 3-4 years and 56 years) and adults. However, they reported that, unlike adults, the children were not influenced by overlap in the identity of the prime and target verb: Only the adults showed a substantially larger priming effect when there was verb overlap. So, although abstract priming effects were evident from early in development, the lexical boost did not emerge until relatively late. These results suggest that it may be possible to use structural priming to look at the way in which the relationship between syntactic representations and the verb lexicon develops.

In adult studies, the relationship between syntactic structure and the verb lexicon has been examined by looking at the effect of verb bias on structural priming. Although many dative verbs can occur in both double object (DOD) and prepositional object datives (PD) (e.g., I gave him a cake/I gave a cake to him), they tend to occur more often in one structure than another (e.g., give tends to occur more often in double object than prepositional object dative structures; see Campbell \& Tomasello, 2001; Gries \& Stefanowitsch, 2004). These preferred argument structure constraints (or verb biases) have a significant effect on adults' choice of syntactic structure in priming studies. For example, in a corpus analysis of English dative verbs, Gries (2005) found that target verbs that were strongly biased towards one structure resisted being primed into another structure. In another study by Coyle and Kaschak (2008), priming effects were found to be larger when the target verb was not strongly associated with one structure (i.e., when it was equi-biased). In other words, an adult's knowledge of a verb's preferred argument structure (e.g., whether this verb occurs more often in a DOD or a PD structure) influences how easy it is to prime that adult to produce that verb in that structure. These verb bias effects provide us with a way of tapping into a participant's knowledge of the links between verbs in the lexicon and syntactic structure.

Another source of verb bias effects is the influence of the prime verb's bias on priming. The identity of the prime verb plays an important role in the size of the priming effect, such that priming is stronger when the verb occurs in a prime structure that is unexpected. For example, Jaeger and Snider (2013) found stronger priming when DOD-biased prime verbs were presented in PD prime structures, and Bernolet and Hartsuiker (2010) reported stronger priming when primes with PD-biased verbs were presented in DOD-structures in Dutch. Fine and Jaeger (2013) reanalysed Thothathiri and Snedeker's (2008b) comprehension study and found that prime structures that were more surprising led to stronger expectations that that same structure would also be used in the target sentence. Jaeger and Snider referred to this as prime surprisal since participants were more likely to be primed when the co-occurrence of the prime verb and prime structure was unexpected. Not only do these results show that adult speakers store information about verbs' syntactic preferences, they also suggest that adults make predictions about prime sentences based on their knowledge of these preferences: When these predictions are not met (i.e., when a verb is presented in an unexpected structure), prime surprisal works to boost the priming effect (Chang, Dell, \& Bock, 2006).

The first aim of the present study then, was to use a structural priming task to determine when children first 
demonstrate knowledge of verb argument structure. To do this we tested the ability of 3-4 year olds, 5-6 year olds and adults to use verb-structure links in a priming production task in three ways; by manipulating (1) verb overlap between prime and target (lexical boost), (2) prime verb bias and (3) target verb bias. If children, like adults, have knowledge of verb argument structure their performance in a priming task should be influenced by the presence or absence of these three factors.

The second aim of the study was to examine the nature of the mechanism that underlies the development of verbstructure links. There are two possibilities that are supported by computational, behavioural, and biological evidence. One is the error-based learning mechanism (Rumelhart, Hinton, \& Williams, 1986) implemented in Chang et al.'s (2006) Dual-Path model, in which the mismatch between the model's predicted sentence and the actual sentence (the error) is used to adjust the model's verb-structure connection weights. The other, is an associative learning mechanism whereby the co-activation of a particular verb and structure strengthens the connection between that verb and that structure.

Both error-based learning and associative learning mechanisms can potentially explain how verb-structure links develop, and we will discuss each of these mechanisms in turn.

\section{Error-based learning mechanisms and structural priming}

The error-based learning account has been applied to syntax acquisition and structural priming in a connectionist model called the Dual-Path model (Chang et al., 2006). On this account, syntax learning occurs because the syntax system generates a prediction about the next word and calculates the difference between the predicted word and the actual word. This difference, or error, is used to make small changes in the weights that support syntactic knowledge in the system. These weight changes gradually converge on the representations that support adult-like sentence production. The model is tested for priming by presenting the prime sentence with error-based learning left ON. The prediction error for the prime is used to make changes to the weights in the network - some of which are made to the model's abstract structural representations. These weight changes persist and influence the model's description of the target, increasing the use of the same structure and thus, creating a structural priming effect.

The development of verb-structure links is explained by the same error-based learning mechanism in the Dual-Path model. Since the model generates predictions and alters syntactic representations on the basis of individual words in sentences, these weight changes enable the model to learn lexical (verb)-structure links at the same time as syntactic structure (see Twomey, Chang, \& Ambridge, 2014, for a version of the model that gradually learned verb biases over development). Thus, verb-structure links are learnt in parallel with knowledge of abstract syntactic structure. The Dual-Path model makes particular predictions about the development of verb-structure links, and how they affect performance in structural priming tasks. First, the account predicts that children will show abstract structural priming as soon as they have acquired abstract structures. Second, because this model predicts that verb-structure links are built by the same mechanism that learns abstract syntactic structure, children should show target verb bias and prime surprisal effects as soon as they demonstrate knowledge of abstract structural priming (i.e., at or soon after the age of three years, Rowland et al., 2012).

However, on this model, the lexical boost is governed by a different cognitive mechanism: explicit memory (Bock \& Griffin, 2000; Chang, Janciauskas, \& Fitz, 2012; Chang et al., 2006). According to this account, the target verb is a cue in the retrieval of an explicit memory of the prime verb and structure; this can explain why adults do not always show lexical boost effects in priming paradigms that reduce the time for explicit processing (e.g., RSVP; Tooley \& Bock, 2014). Given that explicit memory increases with age (Naito, 1990; Sprondel, Kipp, \& Mecklinger, 2011), the boost is predicted to increase in line with the ability to form, store, and retrieve explicit memories. In other words, the model predicts that structural priming, verb bias and prime surprisal effects, but not the lexical boost, will be present from the age of three years.

\section{Associative learning mechanisms and structural priming}

In order to be explicit about what we mean by associative learning, we will focus on a type of associative learning called Hebbian learning (Munakata \& Pfaffly, 2004). According to Hebb (1949), when one neuron A excites another neuron $B$ (and as long as A is excited just before $B)$, the strength of the connection weight between these neurons increases. In this way, persistent exposure to a particular pattern of activation in the input reinforces the responsiveness of a particular output neuron in the future. This type of learning is biologically plausible because it only depends on local changes to pairs of neurons, and the phenomenon of long-term potentiation is a biological instantiation of this mechanism (Bliss \& Collingridge, 1993). A key feature of an associative learning account that models the development of verb-structure links is that changes in the strength of these links are driven solely by the input and are not sensitive to the strength of the weights. Put simply, this means that the amount by which a link is strengthened in response to new input is not affected by previous strengthening of this link. This is in contrast to error-based learning, where weight changes in the model are only made when the model generates an incorrect prediction. Pertinent to the present work, is the fact that Hebbian learning has been used to explain a wide range of empirical findings from the language acquisition literature. For example, the learning of word-concept links is a prototypical associative learning phenomenon, and there are many models that use Hebbian learning mechanisms to simulate this process; Samuelson (2002) used Contrastive Hebbian Learning to train a model to learn the relationship between referents, word forms, and syntactic information, and Li, Farkas, and MacWhinney (2004) used a self-organising Hebbian mechanism to model how we could learn the links between lexical items and semantics. More recently, McMurray, Horst, and 
Samuelson (2012) showed that a model with Hebbian learning combined with dynamic referent selection can explain a wide range of developmental behavioural findings, such as fast mapping and the vocabulary spurt (i.e., an increase in the rate of vocabulary development).

Associative learning can also be used to explain how children develop verb-structure links (see Alishahi \& Stevenson, 2008; Perfors, Tenenbaum, \& Wonnacott, 2010, who instantiate this approach to learning the relation between verbs and structures in computational models). On this account, verbs and abstract structural representations are present from early in development, as is suggested by the abstract structural priming effects shown in the youngest children in Rowland et al. (2012). Each time a verb is heard with a particular syntactic structure (e.g., the dative), the link between that verb and that structure is strengthened by a fixed amount. The development of verb biases (verb argument structure) occurs because of the accumulation of multiple verb-structure experiences to create a bias for one structure. Verb bias and lexical boost effects result from the same associative learning mechanism. On this mechanism, an existing verb-structure link is strengthened after a single prime trial (consistent with adult models of structural priming in which Hebbian association mechanisms are the basis for the lexical boost effect, Reitter, Keller, \& Moore, 2011).

The associative learning model makes a number of predictions about the development of verb-structure links, and how they affect performance in structural priming tasks. Like the error-based learning account, the associative learning account predicts that verb-structure links will grow with development. However, unlike the errorbased learning account, it predicts that the lexical boost and verb bias will develop in parallel because they both stem from the same verb-structure links. In other words, on this account, if three year olds show lexical boost effects, then they should also show target verb bias effects. Contrarywise, if three year olds do not show lexical boost effects (as in Rowland et al., 2012), then they should not show target verb bias effects. Finally, an important feature of the associative learning account is that priming is independent of the strength of verb-structure links. This means that matching and mismatching conditions should prime to a similar extent. For example, if the prime has a PD structure, then the likelihood of also producing a target with a PD structure will increase by some amount (e.g., $5 \%$ ). However, since the size of this priming effect is not influenced by the prime verb's bias, the increase in PD production for a PD prime with a DOD-biased verb will be the same for a PD prime with a PD-biased verb (e.g., 5\%). This is different from the error-based learning account, where priming is stronger when the prime verb's bias mismatches the prime structure (e.g., a DOD-biased verb in a PD structure might increase PD production by $7 \%$ ). Thus, while the error-based learning account predicts prime surprisal, the associative learning account does not.

\section{The current study}

In this study, we used a structural priming paradigm to investigate when children develop adult-like verb-struc- ture links, and to examine two mechanisms, associative and error-based learning, that have been proposed to explain how these verb-structure links are learned. On the error-based learning account, we should see abstract structural priming, verb bias, and prime surprisal effects from an early age, but the lexical boost should increase with age since this has its locus in a separate mechanism. On the associative learning account, we may also see abstract structural priming from an early age, but crucially, the lexical boost and verb bias effects should develop in parallel, because they both stem from the development of verb-structure links. In addition, we should not see prime surprisal effects because priming is independent of the strength of verb-structure links.

The current study used a modified version of the bingo game priming paradigm used by Rowland et al. (2012) to test for structural and verb-specific priming effects in young children (3-4 years), older children (5-6 years), and adults. First, we assessed whether we could replicate the findings of Rowland et al. by examining structural priming and the lexical boost across development. Second, we tested whether children, like adults, would show verb bias effects in priming tasks by exploring whether the size of the priming effect was influenced by the bias of the target verb. Third, we assessed whether children, like adults, would show evidence of prime surprisal. To do this we explored whether the priming effect was stronger when there was a mismatch between prime verb bias and prime syntactic structure (e.g., DOD-biased verb in a PD structure).

\section{Method}

\section{Participants}

A total of 183 participants were tested. One hundred and twenty-three monolingual English-speaking children were recruited from nurseries and schools in the Liverpool area. Sixty-three of these children (32 female) were between five and six years old (mean age $5 ; 8$, age range $5 ; 0-6 ; 11$ ) and 55 children (33 female) were between three and four years old (mean age $4 ; 0$, age range $3 ; 0-4 ; 11$ ). An additional five children from the 3-4 year old age group were tested but produced eight or more (over half) 'other' responses during the task and so were excluded from the final analysis. A further 60 monolingual English-speaking adults (42 female) were recruited from the University of Liverpool student participation pool. Participants were tested individually in either their nursery/classroom or in the language development laboratory at the University of Liverpool.

\section{Design and materials}

\section{Design}

The study used a $3 \times 2 \times 2 \times 2 \times 2$ mixed design. Age (3-4 year olds/5-6 year old/Adults) was the between-subjects variable. ${ }^{1}$ The four within-subjects variables were

\footnotetext{
${ }^{1}$ Although descriptive analyses are presented by age-group, age was coded as a continuous variable. The 3-4 year olds, 5-6 year olds, and Adults were coded as $3.5,5.5$, and 20 years, respectively and then centred.
} 
Prime Type (DOD and PD), Verb Match (Different verb and Same verb condition), Prime Bias (DOD- and PD-biased verbs) and Target Bias (DOD- and PD-biased verbs). The dependent variable was the proportion of dative responses that were DODs (i.e., a ratio of DOD responses over the sum of DOD and PD target responses).

\section{Visual stimuli}

Sixty-four video cartoon animations were created in Anime Studio Pro and were presented in E-Prime 2.0. The cartoons included three pairs of donor and recipient characters that are familiar to young British children and have proper noun names: Tigger and Piglet, Dora (the Explorer) and Boots, and Bob (the Builder) and Wendy. A further three pairs of donor and recipient characters were referred to with determiner + noun NPs: the prince and the princess, the king and the queen, the boy and the girl. Donor and recipient characters were always paired together (e.g., Wendy was always paired with $B o b$, and the prince was always paired with the princess). A further five characters acted as objects and were referred to with non-definite determiner + noun NPs: a cat, a baby, a fish, a puppy, a rabbit. All of the characters were animate to prevent animacy contrasts between the object and recipient influencing syntactic structure, since DOD sentences tend to occur with animate recipients and inanimate objects.

Thirty-two of the animations depicted transfer actions that can be described with dative sentences. Thirty-two others were used as fillers and depicted non-causal actions that can be described with intransitive sentences. Eight of the animations that were used as fillers were also used in a practice session. Each prime picture was always paired with a target picture that included different characters from those in the prime. Animations also depicted the direction of motion of transfer actions equally often from right-to-left and from left-to-right to control for the possibility that direction of transfer would influence structure choice.

Bingo cards were created to match each video cartoon animation. Four bingo boards were created on which to place the cards during the 'game'. Two of these boards included a grid of four squares and were used in a practice session before the actual experiment. The other two boards were used in the experiment and contained nine squares.

\section{Sentence stimuli}

The four verbs used - give, show, bring, and send - are alternating dative verbs that are familiar to young children. Analysis of the Manchester corpus (available from the CHILDES database, MacWhinney, 2000), which contains the spontaneous speech of 12 English-speaking children and their mothers, revealed that the verbs give and show occurred more frequently in DOD structures (78\% and $80 \%$ of the time, respectively). These verbs were therefore considered to be DOD-biased. Bring and send were found to occur more often in PD structures $(63 \%$ and $74 \%$ of the time, respectively), so these verbs were classified as PDbiased. Similar verb-syntactic preferences have been reported in other work (e.g., Campbell \& Tomasello, 2001; Gropen, Pinker, Hollander, Goldberg, \& Wilson, 1989).
Ninety-six different sentences, including 32 verb-stems, were created to describe the 64 video cartoon animations. Thirty-two of these sentences described thirty-two different cartoon animations (eight DOD sentences for each verb). These were used as primes and depicted transfer actions using a DOD structure (e.g., Dora gave Boots a rabbit). A further 32 prime sentences described the same transfer actions but used the PD structure (e.g., Dora gave a rabbit to Boots). Thirty-two target verb-stems (eight verb-stems for each verb) were created (e.g., The boy brought...) in addition to 32 filler sentences, which used an intransitive structure (e.g., The princess jumped).

Each verb was presented eight times per participant: four times in the prime sentence (twice in the PD structure and twice in the DOD structure) and four times in the target verb-stem. Each participant was exposed to 16 primetarget pairs, which alternated with filler-filler pairs to minimise priming effects between pairs. ${ }^{2}$ Overall, each participant was presented with, and produced, 64 sentences in total. No participant was asked to produce the same prime sentence twice and all participants were exposed to an equal number of prime-target pairs from each of the prime conditions.

We explored both lexically-specific and lexically-independent priming as a within-subjects variable so participants were exposed to sentences in which verbs were repeated across primes and targets and also sentences where the verbs in primes and targets were different. Pairs of characters appeared equally often in prime and target sentences and, to avoid lexical overlap (other than that of the verb), characters in primes were always different from the characters in the targets with which they were paired. Furthermore, primes that contained determiner noun phrases (e.g., the princess) were always followed by targets with proper noun phrases (e.g., Wendy), and vice versa, to limit the possibility that participants would be primed by the prosody of the prime sentence. Additionally, sentences were always presented in the past tense to avoid repetition of the regular progressive (-ing) ending. Three of the verbs were irregular (gave, sent, brought) to avoid repetition of the regular past tense (-ed) ending.

To control for sentence-specific preferences, 12 counterbalance groups were created to ensure that: (1) sentences that appeared as a DOD prime in one counterbalance group appeared as a PD prime in another, and vice versa; (2) all characters in both prime and target sentences appeared equally often with each verb, and (3) each prime verb was paired with itself and the three remaining verbs equally often in target sentences. All sentences across counterbalanced groups were presented semi-randomly to ensure that participants could not predict the structure of consecutive prime sentences. This also enabled us to ensure that characters appearing in a fillerfiller pair had not appeared in the preceding target sentence or in the following prime sentence.

\footnotetext{
2 One reviewer commented that the alternation of prime-target pairs with filler-filler pairs may have made the structure of the experiment obvious to the participants. However, due to the complex and interactive nature of the task none of the children or adults, when asked, correctly guessed the aim of the study.
} 


\section{Procedure}

\section{Children}

The experiment used a paradigm adapted from Rowland et al. (2012), and was conducted in the form of a bingo game in which the experimenter and the child took turns to describe cartoon animations on a laptop computer to a confederate. The experimenter introduced all of the characters involved in the task to the child by showing them a selection of bingo cards on which these characters appeared. They then sat in front of the computer side by side, whilst the confederate sat opposite. The experimenter described the cartoon on the left-hand side of the screen (the prime sentence) and asked the child to repeat the prime sentence, addressing a hand puppet held by the confederate. The child was then asked to produce a target sentence by describing a cartoon animation on the right-hand side of the screen. A stem-completion technique was used to ensure that the child's response contained the target verb, although the child was encouraged to produce responses in the form of whole sentences. For example, the experimenter would say "Wendy showed...". The child would then complete the description of the animation using this target stem (e.g., either, "Wendy showed a rabbit to Bob" or "Wendy showed Bob a rabbit"). After each sentence, the confederate looked to see if he/she had the bingo card corresponding to that cartoon. If he/she did, the correct bingo card was given to the experimenter or child as appropriate.

Each dative prime-target pair was immediately followed by an intransitive filler-filler pair. The first person to fill the bingo grid with bingo cards was the winner of the game and the experiment was designed so that the participant always won. Before running the experiment using the nine-squared bingo board, a practice session using the four-squared board was carried out to ensure that the children understood the task.

\section{Adults}

The procedure for adult participants was identical to the procedure for child participants except that: (a) adults were told that we were investigating how well children could describe a variety of scenes using different words and that we needed adults as a comparison group, (b) adults received explicit instructions to repeat the prime and to produce responses in the form of full sentences, and (c) adults did not have to direct their speech towards a hand puppet.

\section{Coding}

Target responses were recorded online by the experimenter using the keyboard response coding function of E-Prime 2.0 (the experimenter pressed ' $\mathrm{p}$ ', ' $\mathrm{d}$ ', or 'o' depending on whether the participant produced a PD, DOD, or 'other' response, respectively, and these responses were automatically recorded and collated into a data file by E-Prime). The experiment was also audiotaped, allowing the transcription and coding of the utterances off-line by the first author. A second coder rated $10 \%$ of the utter- ances, and Cohen's (1960) kappa revealed very good interrater reliability: $\kappa=0.97$ (Landis \& Koch, 1977).

Many of the young children and some of the older children needed prompting by the experimenter to produce the prime and the entire target sentence correctly. Some of them, however, only produced partial target responses (e.g., they completed the stem without including the target verb). In order to capture these partial target responses, we employed three levels of coding: lax, intermediate, and strict. To qualify for lax coding, the prime sentence had to be repeated correctly but the participant might have received help to do this. In addition, the participant may not have produced the target verb or the entire target utterance, but may have instead just completed the target stem. To qualify for intermediate coding, the prime sentence had to be repeated correctly with the participant needing minimal help to do this. In addition, the entire target utterance was produced, but prompting to do this may have been needed more than once. To qualify for strict coding, the prime and target sentence had to be produced correctly with no more than one prompt. A target response was considered a DOD if it contained the correct target verb followed by two noun phrases, and a PD if it contained the correct target verb followed by a noun phrase and a prepositional phrase headed by 'to'. Responses coded as 'other' were those where: (a) the participant failed to repeat the prime correctly (even after help), (b) the participant produced a non-target verb and, (c) the target sentence included the preposition 'at' rather than 'to'. Preliminary analysis revealed that all of the coding schemes generated very similar patterns of results, and so the following analyses are reported only on the strictly-coded data.

\section{Results}

In this study we wanted to: (1) assess the relation between structural priming and the lexical boost across development, (2) explore whether the bias of the target verb influenced structural priming across development, and (3) investigate whether a mismatch between prime verb bias and prime structure (prime surprisal) influenced structural priming across development.

A variety of logistic mixed effect models were fitted to examine our data (Baayen, Davidson, \& Bates, 2008; Jaeger, 2008). All of the models were calculated using the glmer function of the lme4 package in $\mathrm{R}$ (lme4: version 1.1-6; R Core Team, 2012). In all cases, the dependent measure was the production of double object dative structures $(\mathrm{DOD}=1, \mathrm{PD}=0)$. All factors were effect/sum coded (Wendorf, 2004), except for age in years, which was centred to reduce multi-collinearity (Neter, Wasserman, \& Kutner, 1985). Maximal models were fitted and the random slope structure was simplified until the model con-

\footnotetext{
3 Under the lax coding scheme, 3-4-year olds, 5-6-year olds, and adults produced $13 \%, 3 \%$, and $1 \%$ 'other' responses, respectively. Under the intermediate coding scheme, 3-4-year olds, 5-6-year olds, and adults produced $22 \%, 5 \%$, and $3 \%$ 'other' responses respectively. Under the strict coding scheme, 3-4-year olds, 5-6-year olds, and adults produced $24 \%, 7 \%$, and $3 \%$ 'other' responses, respectively.
} 
verged following the procedure in Barr, Levy, Scheepers, and Tily (2013). Model comparison was used to compute chi-square and $p$-values.

\section{Structural priming, the lexical boost, and target verb bias across development}

Analysis 1 tested whether we could replicate the findings of Rowland et al. by examining structural priming and the lexical boost across development (aim 1), and whether the size of the priming effect was influenced by the bias of the target verb (aim 2). Fig. 1 shows the mean proportion of DOD responses after DOD and PD primes in the Same verb and the Different verb conditions for each age group. Structural priming was demonstrated if there was a greater proportion of DOD responses after DOD primes than after PD primes. A lexical boost was demonstrated if there was a bigger priming effect in the Same verb condition than in the Different verb condition. Target verb bias was demonstrated if there was a higher proportion of DOD target responses with DOD-biased verbs, and a lower proportion with PD-biased verbs. Fig. 2 shows the same data as Fig. 1 divided by Target Bias (DODbiased/PD-biased).

Our first model included as fixed effects: (a) Age (34 year olds/5-6 year old/Adults); (b) Prime Type (DOD/ PD); (c) Verb Match (Different verb/Same verb); and (d) Target Bias (DOD-biased verb/PD-biased verb). The model included by-subject random slopes for Target Bias. The results revealed a main effect of Prime Type $(\beta=1.42$, $\left.\chi^{2}(1)=184, p<.001\right)$, indicating that there was a structural priming effect; the participants produced more DOD responses after DOD primes than after PD primes. A main effect of Age $\left(\beta=0.13, \chi^{2}(1)=29.0, p<.001\right)$ indicated that the likelihood of producing DOD responses overall increased with age. In addition, an interaction between Age and Prime Type $\left(\beta=0.11, \chi^{2}(1)=39.3, \quad p<.001\right)$ showed that, overall, the size of the priming effect increased with age. There was no interaction between Prime Type and Verb Match, but there was a three-way interaction between Age, Prime Type, and Verb Match $\left(\beta=0.15, \chi^{2}(1)=21.4, p<.001\right)$. The positive beta indicates that the lexical boost grew over development.

With respect to target verb bias (Fig. 2), a main effect of Target Bias $\left(\beta=1.19, \chi^{2}(1)=75.0, p<.001\right)$ indicated that participants were more likely to produce DODs with DOD-biased target verbs than with PD-biased target verbs. There was also a three-way interaction between Age, Verb Match, and Target Bias $\left(\beta=-0.08, \chi^{2}(1)=6.18, p<.05\right)$, indicating that the effect of target verb bias grew with age and that it differed across verb match conditions. Importantly, however, there was no interaction between Target Bias and Prime Type, indicating that, although the bias of the target verb affected which structure the participants produced overall, it did not affect the size of the priming effect.

To explore both of the three-way interactions produced by the model, we fitted separate models to each age group. We also calculated effect sizes (Cohen's $d$ ) to allow direct comparison of groups while controlling for differences in sample size and variance (factoring out shared variation, as in Dunlap, Cortina, Vaslow, \& Burke, 1996). Three separate models were run on each age group, and all models included by-subject random slopes for Target Bias. We will first review the results from each model for priming and the lexical boost to explore the interaction between Age, Prime Type, and Verb Match (Table 1). We will then separately review the results for Target Bias to explore the interaction between Age, Verb Match, and Target Bias (Table 2).

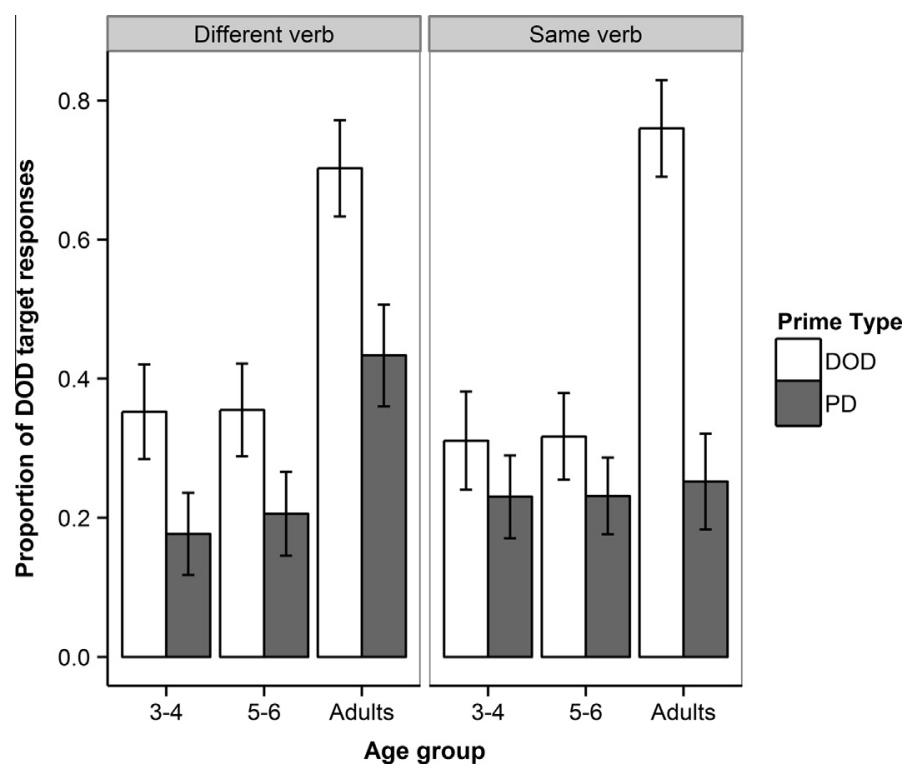

Fig. 1. Mean proportion of DOD responses after DOD and PD primes when primes and targets were different (Different verb) and the same (Same verb) (standard error bars generated with remef; Hohenstein \& Kliegl, 2013). 

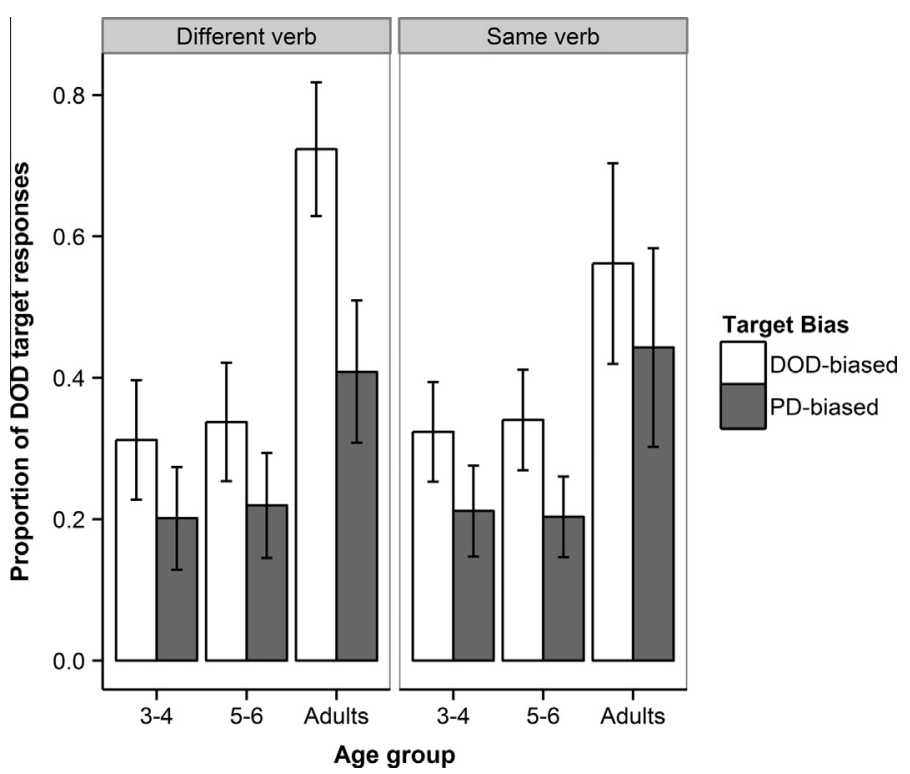

Fig. 2. Mean proportion of DOD responses in the Different and Same verb condition with DOD- and PD-biased target verbs (standard error bars generated with remef; Hohenstein \& Kliegl, 2013).

Table 1

Size of priming effect in the Different verb and Same verb conditions - calculated both as the proportion of DODs produced in each prime condition (difference score) and as effect sizes (Cohen's $d$ ).

\begin{tabular}{|c|c|c|c|c|c|c|}
\hline \multirow[t]{2}{*}{ Age } & \multicolumn{3}{|l|}{ Different verb } & \multicolumn{3}{|l|}{ Same verb } \\
\hline & Difference score & Standard error & Cohen's $d$ & Difference score & Standard error & Cohen's $d$ \\
\hline $3-4$ & 0.15 & 0.049 & 0.45 & 0.03 & 0.041 & 0.08 \\
\hline $5-6$ & 0.14 & 0.030 & 0.42 & 0.06 & 0.023 & 0.17 \\
\hline Adults & 0.27 & 0.048 & 0.90 & 0.50 & 0.044 & 1.86 \\
\hline
\end{tabular}

Table 2

Size of target verb bias effect in the Different verb and Same verb conditions - calculated both as the proportion of DODs produced with DOD-biased target verbs minus the proportion of DODs produced with PD-biased target verbs (difference score) and as effect sizes (Cohen's $d$ ).

\begin{tabular}{|c|c|c|c|c|c|c|}
\hline \multirow[t]{2}{*}{ Age } & \multicolumn{3}{|l|}{ Different verb } & \multicolumn{3}{|l|}{ Same verb } \\
\hline & Difference score & Standard error & Cohen's $d$ & Difference score & Standard error & Cohen's $d$ \\
\hline $3-4$ & 0.11 & 0.043 & 0.39 & 0.11 & 0.051 & 0.33 \\
\hline $5-6$ & 0.12 & 0.035 & 0.33 & 0.14 & 0.028 & 0.39 \\
\hline Adults & 0.31 & 0.037 & 1.18 & 0.12 & 0.035 & 0.46 \\
\hline
\end{tabular}

Table 1 shows the size of the priming effect by age group and verb match condition calculated both as a difference score (\% DOD responses produced after DOD primes minus \% DOD responses produced after PD primes) and as Cohen's $d$. The 3-4 year olds showed a main effect of Prime Type $\left(\beta=0.85, \chi^{2}(1)=14.2, p<.001\right)$, but no effect of Verb Match and no interaction between Prime Type and Verb Match. This means that the 3-4 year olds showed structural priming effects, but showed no lexical boost. Comparison of the effect sizes confirmed this interpretation; in fact, the effect size (Cohen's $d$ ) was smaller (not larger) in the Same verb (0.08) than in the Different verb condition (0.45). The data from the 5-6 year olds showed a similar pattern. There was a main effect of Prime Type $\left(\beta=0.98, \chi^{2}(1)=21.6, p<.001\right)$, but no effect of Verb
Match, and no interaction between Prime Type and Verb Match. Once again, comparison of the effect sizes confirmed this interpretation; there was structural priming but no lexical boost. The adults' data revealed a main effect of Prime Type $\left(\beta=2.25, \chi^{2}(1)=179.0, p<.001\right)$ and a main effect of Verb Match $\left(\beta=-0.42, \chi^{2}(1)=6.03, p<.01\right)$. In contrast to the children however, there was a significant interaction between Prime Type and Verb Match $\left(\beta=1.29, \chi^{2}(1)=19.2, p<.001\right)$, indicating that the size of the priming effect was bigger in the Same verb condition than in the Different verb condition. The comparison of effect sizes confirmed this interpretation; Verb overlap in the Same verb condition boosted the structural priming effect (by 23\%). Thus, unlike the children, there was a large lexical boost effect in the adults. 
Table 2 shows the size of the target verb bias effect by age group and verb match condition calculated both as a difference score and as Cohen's $d$.

There was a main effect of Target Bias in all age groups; 3-4 year olds $\left(\beta=1.09, \chi^{2}(1)=10.5, p<.01\right), 5-6$ year olds $\left(\beta=1.40, \quad \chi^{2}(1)=24.9, \quad p<.001\right)$ and adults $(\beta=1.35$, $\left.\chi^{2}(1)=41.5, p<.001\right)$. In other words, all age groups were influenced by the bias of the target verb, producing more DOD responses with DOD-biased target verbs than with PD-biased target verbs. There were no other main effects or interactions for the children, confirmed by the effect sizes, which are similar across the Same and Different verb conditions for both 3-4 and 5-6 year olds. However, the adults showed a significant interaction between Verb Match and Target Bias $\left(\beta=-1.08, \chi^{2}(1)=9.76, p<.01\right)$, indicating that the adults matched target verb bias less often in the Same verb condition than in the Different verb condition. Comparison of the effect sizes confirmed this interpretation; the effect size (Cohen's $d$ ) was smaller in the Same verb condition $(0.46)$ than in the Different verb condition (1.18). In other words, in the Same verb condition (presumably because the lexical boost dominated structural choice), the adults were less likely to default to matching the target verb biases. Thus, the three-way interaction of Age, Verb Match, and Target Bias shows that target verb bias increased over development, but that this effect was mediated by the lexical boost in the Same verb condition for the adults.

\section{Prime surprisal across development}

Our third aim was to investigate whether children and adults were sensitive to prime surprisal. For this analysis, we created a Prime Bias Match variable. This allowed us to test whether the priming effect was larger when the prime verb bias mismatched the prime structure (e.g., Mismatch $=$ a PD-biased verb in a DOD structure) compared to when the prime verb bias matched the prime structure (e.g., Match $=$ a DOD-biased verb in a DOD structure). Analyses were conducted on the Different verb condition data only. This was to ensure that any difference in the priming effect between "Match" and "Mismatch" conditions was isolated to an effect of the prime sentence, and was not confounded by lexical boost effects. For example, the "Mismatch" sentences in the Same verb condition used the same prime and target verb. Thus, an increase in priming after sentences may have been interpreted as prime surprisal, when actually this boost in priming was a consequence of verb repetition, and vice versa. The model included as fixed effects: (a) Age (3-4 year olds/5-6 year old/Adults), (b) Prime Type (DOD/PD), (c) Prime Bias Match (Match/Mismatch), and (d) Target Bias (DOD-biased/PD-biased). It included by-subject random slopes for Prime Type and Target Bias.

We replicated many of the main effects and interactions we saw in analysis 1 . There was a main effect of Prime Type $\left(\beta=1.42, \chi^{2}(1)=32.6, \quad p<.001\right)$, a main effect of Age $\left(\beta=0.14, \chi^{2}(1)=18.0, p<.001\right)$, and a main effect of Target Bias $\left(\beta=1.28, \chi^{2}(1)=63.2, p<.001\right)$. There was also a sig- nificant interaction between Age and Target Bias $\left(\beta=0.10, \chi^{2}(1)=11.1, p<.001\right)$, with the positive beta again indicating that verb bias effects became stronger with age. However, there was no interaction between Prime Type and Target Bias, or between Prime Bias Match and Target Bias. This indicates that the bias of the target verb did not interact with the priming effect (as in analysis 1 ) or with the effect of prime surprisal.

Importantly, there was a three-way interaction between Age, Prime Type, and Prime Bias Match $\left(\beta=-0.10, \chi^{2}(1)=4.19, p<.05\right)$. The negative beta for this interaction indicates that priming was stronger in the Mismatch condition compared to the Match condition, and that this difference decreased with age (see Fig. 3). No other interactions reached significance.

To explore the significant interaction between Age, Prime Type, and Prime Bias Match in more detail, we fitted separate models to each age group. As in analysis 1 , we also calculated effect sizes (Cohen's $d$ ) to allow direct comparison of groups while controlling for differences in sample size and variance. Since Target Bias did not interact with any of the priming effects in the main analysis, we collapsed across Target Bias. All models included by-subject random slopes for Prime Type. Table 3 reports the size of the priming effect for each age group and verb condition calculated both as a difference score and as Cohen's $d$.

All age groups showed main effects of Prime Type, indicating that all ages were primed (as in analysis 1 above; $3-$ 4 year olds; $\beta=1.26, \chi^{2}(1)=7.92, p<.01 ; 5-6$ year olds: $\beta=0.95, \chi^{2}(1)=8.29, p<.01$; adults: $\beta=1.38, \chi^{2}(1)=27.2$, $p<.001$ ). Both groups of children also showed a significant interaction between Prime Type and Prime Bias Match, indicating that there was significantly more priming in the Mismatch condition than in the Match condition (i.e. a prime surprisal effect; $3-4$ year olds: $\beta=1.33, \chi^{2}(1)=4.58$, $p<.05$; 5 -6 year olds: $\left.\beta=1.18, \chi^{2}(1)=5.24, p<.05\right)$. This was confirmed by the effect size analysis: the effect sizes in the Mismatch condition were more than double the size of the effect sizes in the Match condition for both the 34 year olds (Cohen's $d$ : Mismatch $=0.61$ vs Match $=0.25$ ) and the 5-6 year olds (Cohen's $d$ : Mismatch $=0.57$ vs Match $=0.15$ ). However, in adults, the difference in effect size was smaller (Cohen's $d$ : Mismatch $=0.85$ vs Match $=0.50$ ), and was only marginally significant $\left(\beta=0.73, \chi^{2}(1)=2.87, p=.091\right)$.

To summarise, as in previous studies (e.g., Rowland et al., 2012), there were significant structural priming effects at all ages, but the size of the lexical boost increased with development. With respect to target verb bias, we found that all groups preferred to produce target sentences with the syntactic structure that matched the bias of the target verb and that this tendency grew over development. This increase in verb bias knowledge over development was not seen in the Same verb condition potentially due to interference from the lexical boost. Finally, we found that priming was stronger when there was a mismatch between the prime verb's bias and its structure (prime surprisal), although the effect was only marginal in the adults. Prime surprisal was strongest in the children and decreased over the course of development. 


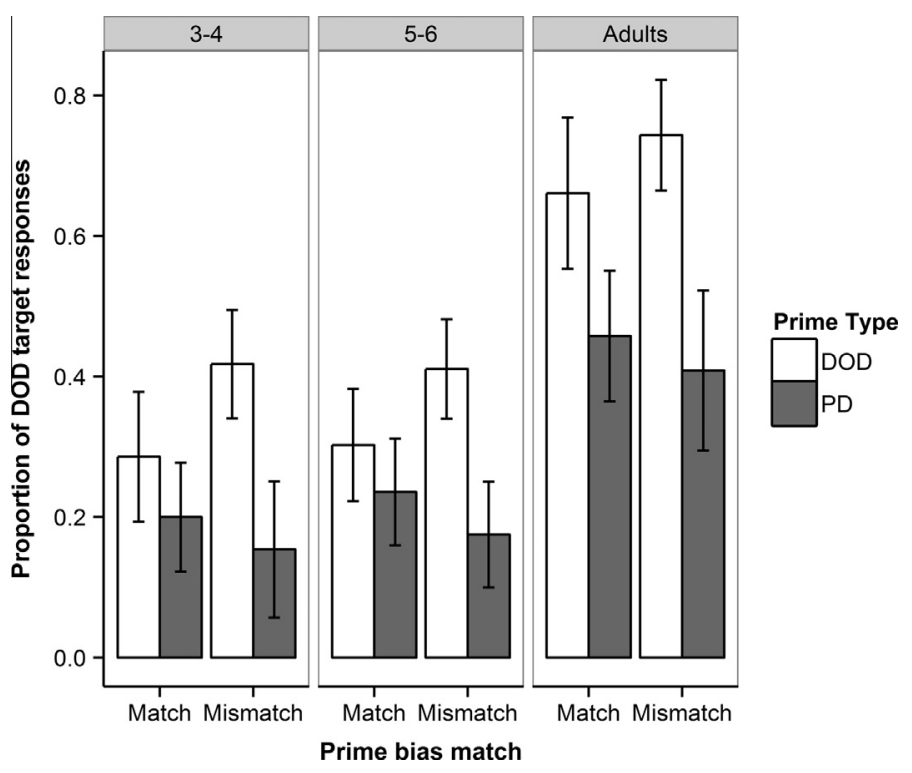

Fig. 3. Mean proportions of DOD responses when prime verb bias matched prime structure (Match), and when prime verb bias mismatched prime structure (Mismatch) (standard error bars generated with remef; Hohenstein \& Kliegl, 2013).

Table 3

Size of priming effect when prime verb bias and structure were the same (Match), and when prime verb bias and prime structure were different (Mismatch) calculated both as the difference between the proportion of DODs produced in each prime condition (difference score) and as effect sizes (Cohen's $d$ ).

\begin{tabular}{|c|c|c|c|c|c|c|}
\hline \multirow[t]{2}{*}{ Age } & \multicolumn{3}{|l|}{ Match } & \multicolumn{3}{|l|}{ Mismatch } \\
\hline & Difference score & Standard error & Cohen's $d$ & Difference score & Standard error & Cohen's $d$ \\
\hline $3-4$ & 0.09 & 0.070 & 0.25 & 0.24 & 0.261 & 0.61 \\
\hline $5-6$ & 0.06 & 0.050 & 0.15 & 0.22 & 0.050 & 0.57 \\
\hline Adults & 0.20 & 0.070 & 0.50 & 0.33 & 0.072 & 0.85 \\
\hline
\end{tabular}

\section{Discussion}

In this study, we used a priming paradigm to investigate when children develop adult-like verb-structure links, and to test two accounts of how children develop knowledge of verb argument structure. On the error-based learning account, knowledge of verb argument structure develops early in development. Thus, we should see target verb bias and prime surprisal effects in all ages at which we see structural priming effects (i.e. from three years of age, Rowland et al., 2012). However, the lexical boost, which is attributed to a separate explicit memory system, should develop with age. The associative learning account treats the lexical boost and verb bias as the result of the same Hebbian learning mechanism. Thus, it predicts that the lexical boost and verb bias effects will develop in parallel; if three-year olds show lexical boost effects, they should also show target verb bias effects, but if they do not show lexical boost effects (as in Rowland et al., 2012), they should also not show target verb bias effects. In addition, priming on this account is independent of the strength of verbstructure links. Thus, unlike the error-based learning account, on the associative learning account, three-year olds should not show evidence of prime surprisal effects.
We first assessed whether we could replicate the findings of Rowland et al., which showed that structural priming effects were present across development, but that the lexical boost grew with age. Second, we tested whether children, like adults, were influenced by the argument structure bias of the target verb. Third, we examined whether children, like adults, were sensitive to prime surprisal.

We replicated the findings of Rowland et al., finding evidence for structural priming across development in that young children, older children, and adults were significantly more likely to produce DOD sentences (e.g., Wendy gave Bob a rabbit) after hearing and repeating DOD primes (e.g., The boy sent the girl a fish) than after PD primes (e.g., The boy sent a fish to the girl). These results provide additional support for the idea that, by the age of three, young children have built some form of abstract syntactic representation for the English dative construction that enables them to generalise across similarly-structured sentences (see also Bencini \& Valian, 2008; Messenger, Branigan, McLean, \& Sorace, 2012; Shimpi et al., 2007 for other evidence for early acquired knowledge). Like Rowland et al., we also observed a lexical boost that increased with development: adults showed increased priming (23\%) when the 
verb in the prime matched the verb in the target, but 3-4year old and 5-6-year old children did not. In other words, although structural priming effects were apparent across development from age three to adulthood, the lexical boost was only apparent in adults.

We then tested when children, like adults, first demonstrated evidence that they were influenced by the bias of the target verb. Although target verb bias did not interact with the size of the priming effect for either children or adults, we found that it did affect which structure they produced; all age-groups produced more DOD responses with DOD-biased target verbs (give, show) than with PDbiased target verbs (bring, send). In other words, children as young as three years old already knew, and were influenced by, the alternation biases of the four dative target verbs used in this study, even though they did not exhibit the verb-based lexical boost. This effect of target verb bias, but not lexical boost, in the 3-4 year olds is consistent with the error-based learning account as instantiated in the Dual-Path model (Chang et al., 2006), but not the associative learning mechanism, which predicts a tight relationship between lexical boost and target verb bias throughout development.

Our final aim was to test when children, like adults, first demonstrated evidence of prime surprisal. We manipulated prime surprisal by including verbs with biases that matched or mismatched the prime structure; more priming is predicted when the verb's bias mismatches the structure in which it appears. Again, we found significant effects across all three age groups; 3-4 year olds, 5-6 year olds, and adults all showed stronger priming effects when there was a mismatch between the prime verb's bias and the prime structure (prime surprisal), although the difference was only marginal in the adults. In other words, the bias of the prime verb had an effect on the size of the priming effect, and this was stronger in the children than in the adults. Again, the presence of prime surprisal in the 3-4 and 5-6 year olds, in the absence of a lexical boost is predicted by the error-based learning account, but not the associative learning account.

Surprisal effects are difficult to explain in terms of the associative learning account because it predicts that priming is independent of the strength of verb-structure links (i.e., matching and mismatching conditions should prime to a similar extent). The error-based learning mechanism, however, directly predicts prime surprisal because priming is due to the mismatch in verb-structure prediction. For example, the model might predict that after a PD-biased (prime) verb sequence like, "He sent the...", the next word should be an inanimate word like letter. However, if the prime structure is actually a DOD structure, then the next word is more likely to be an animate word like girl. This mismatch between the predicted next word and the actual next word (error) is used to change the weights in the model so that it better predicts girl after "He sent the...". Since the weights in the model are initially set randomly, the model must learn about verb-syntactic preferences using error-based learning. In the model's equivalent of a three-year old, these preferences are established by this point, and so prime surprisal effects should be seen early in development.
Explaining the developmental pattern of target verb bias and prime surprisal

Overall, our findings support the Dual-Path model which predicts that both abstract syntactic structures and verb-structure links are a product of the same error-based learning mechanism. Structural priming, effects of target verb bias, and effects of prime verb bias (prime surprisal), but not lexical boost effects, were present at all three ages tested. There are, however, two findings in our data that remain to be explained. First, the effect of target verb bias increased over development: adults were more strongly influenced by the bias of the target verb than children. Second, the effect of prime surprisal decreased over development; adults were less influenced by the bias of the prime verb than children. This apparent contradiction is difficult to explain if we assume that both effects come from the same source; the participants' knowledge of a verb's preferred argument structure, which both influences the choice in production of the target and generates the expectation that creates primes surprisal.

We speculate here that it is possible to explain this finding in terms of the dynamics of the learning process. The development of target verb bias can be explained by a gradual accumulation of experience of verbs in their argument structure preferences: A verb's bias must be learned from the sample of verb-structure pairs in the input but, at any one time, the probability of a particular verb-structure pair occurring may not match the cumulative long-term probabilities (flipping a fair coin once will never yield 0.5 heads). Whether we assume that a child's knowledge of a verb's syntactic preference begins with a random initial bias towards one structure (due to random initial connectivity in the brain) or with a fixed initial bias like 0.5 , children should gradually accumulate evidence for the verb biases in the input over development. For example, although the verb throw may have an overall bias towards the PD frame, there may be periods of time in which throw is heard most often in a DOD structure. Early on in the learning process, these instances weaken the PD bias of the representation of the verb throw, which means that in the beginning, syntactic representations are likely to be less stable. Thus, learning verb biases is a gradual process of accumulating evidence over time, which can be temporarily derailed by random fluctuations in the input. This can explain why target verb bias effects are smaller in young children than adults; within any one group of children at any one point in time, there will be substantial individual variation in the strength of the target verb bias, which leads to a smaller group effect. This account is consistent with the gradual development of verb-structure links in the Dual-Path model for the locative alternation (Twomey et al., 2014).

The decrease in prime surprisal over development can be explained by changes in the magnitude of the learning rate, instantiated by a reduction in plasticity in connectionist models (Ellis \& Lambon Ralph, 2000). Reduction in plasticity is important in these models to keep newly learned knowledge from overwriting older knowledge (catastrophic interference; McClelland, McNaughton, \& O'Reilly, 1995); Chang et al. (2006) instantiated this reduc- 


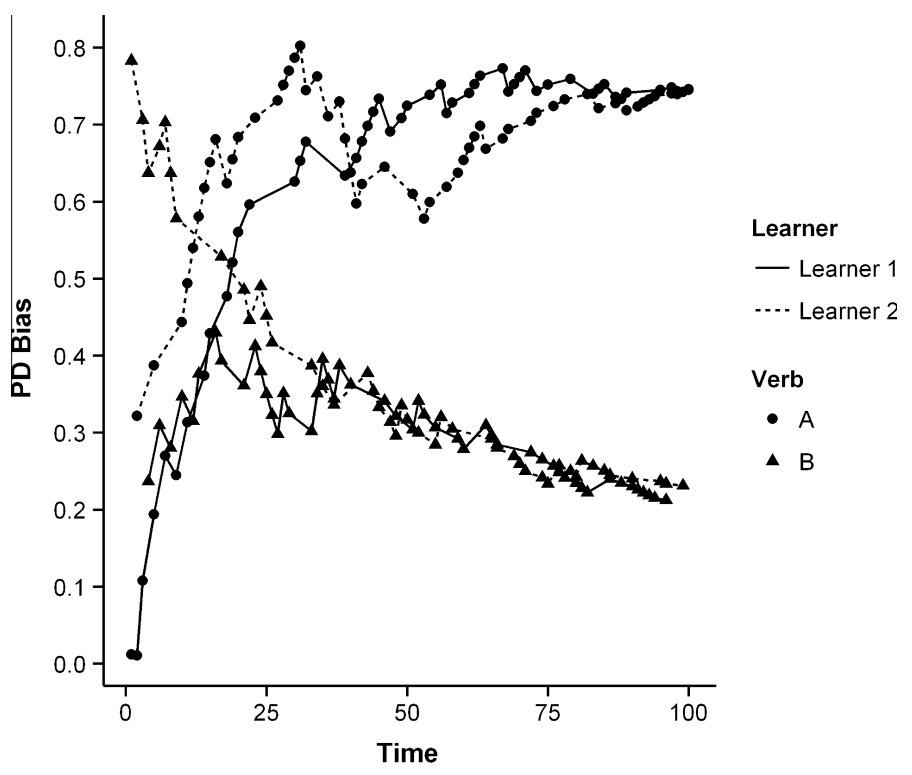

Fig. 4. Development of target verb bias for two verbs (A and B) over 100 exemplars for two hypothetical learners (learner 1 and learner 2).

tion in plasticity by reducing a learning rate parameter over training. This has implications for priming, because in this model, priming involves the same error-based learning mechanism that is used for learning language. Faster learning rates lead to more substantial weight changes in response to error and, thus, more prime surprisal. So, if the learning rate is higher early in development, this means that the effect of surprisal on priming will be larger earlier in development. In other words, we see greater prime surprisal early in development because initial learning is faster than later learning.

To clarify how these two effects could arise within a developmental account of language acquisition, we developed a simple dynamic systems model of verb bias acquisition. We gave the model 100 inputs for two verbs (A, B), which were coded as 1 for PD and 0 for DOD (PDINPUT). Each PDINPUT instance can be thought of as a PD or DOD prime structure and, through learning, long-term expectations for the verb-structure links are strengthened. When these changes accumulate over time and become stable, then we call this the verb's structural bias. To create verb biases, we specified that verb A occurred in a PD $80 \%$ of the time and verb B occurred in a PD $20 \%$ of the time. We also generated two random learners (learner 1 and learner 2). To implement the change in plasticity over development, a learning rate parameter was set to start at 0.1 and reduce to 0.01 in 100 steps. The model is governed by three equations. The first equation (1) implements error-based learning by updating the structural bias for the input verb $\mathrm{V}$. This is done by adjusting the previous bias for that verb by the error between the previous bias and the present input ( $P D=1, \mathrm{DOD}=0$ ), multiplied by the learning rate (this only changes the bias for the input verb; the biases for other verbs remain unchanged). Since it is standard to randomize weights initially in connectionist models (and the links between neurons in the brain are not set initially to some uniform strength), the second equation (2) sets the initial bias to a uniform random number between 0 and 1 . The choice of structure that is produced is assumed to be a function of the bias of the verb that has been chosen (3). These equations were used to generate each random learner:

$$
\begin{aligned}
& \text { BIAS }_{\mathrm{V}, \mathrm{T}}=\text { BIAS }_{\mathrm{V}, \mathrm{T}-1}+\left(\text { PDINPUT }_{\mathrm{T}}-\text { BIAS }_{\mathrm{V}, \mathrm{T}-1}\right) * \mathrm{LRATE}_{\mathrm{T}} \\
& \text { BIAS }_{\mathrm{V}, 0}=\text { UniformRandom }(0,1) \\
& \text { PDCHOICE }_{\mathrm{T}}=\text { BIAS }_{\mathrm{V}, \mathrm{T}} \text { if verb is V }
\end{aligned}
$$

The initial bias (at time 0 ) is a random number between 0 and 1 and hence learner 2 has an initial bias of 0.79 for verb $\mathrm{B}$, which is a bias for the PD. But since verb $\mathrm{B}$ occurs in the PD $20 \%$ on average, the bias gradually decreases over development until the learner matches the adult's baseline bias. On the other hand, verb B in learner 1 is already close to the adult level (0.24, DOD-biased) at the start of development. However, due to a random run of PDs early in development, the bias moves first towards the PD before returning to the adult level.

Fig. 4 shows that, in this model, although early verb biases might not match adult biases, the gradual learning of adult biases can explain the growth of the target bias in our study. The variable learning rate in the figure also explains why prime surprisal effects are larger early in development. The learning rate starts off high and decreases over development, which means that early verb biases are affected more substantially by the prime structure than later verb biases (i.e., vertical displacement is greater). For example, for verb A, learner 2 starts off with a bias of 0.31 but, once the first PD is experienced, the bias increases by 0.08 because the learning rate is high. Later in development, the bias changes by only 0.01 because the learning rate is lower. This explains why prime surprisal effects are larger earlier in development. ${ }^{4}$

\footnotetext{
${ }^{4}$ A faster learning rate implemented in a Hebbian mechanism would lead to more priming in both Match and Mismatch conditions.
} 
The dynamic systems model helps us to understand how a model that allows verb-structure representations to change over development could provide an explicit account of the behavioural changes in this study. Although this is motivated by assumptions in connectionist models of development, it is consistent with the literature on the critical/sensitive period. For example, language learning ability is strongly associated with the age that language learning starts, even when the amount of input is controlled (Johnson \& Newport, 1989; Lenneberg, 1967; Mayberry, 2007), which supports the view that the learning rate may change with age. The fact that critical/sensitive periods exist in many domains (e.g., Crawford, Harwerth, Smith, \& von Noorden, 1996; Daw, 1994; Katz \& Shatz, 1996; Kral \& Sharma, 2012; Wiesel \& Hubel, 1965) suggests that these learning rate changes may be governed by neural mechanisms that are not specific to language learning (e.g., myelination, axon elaboration, synapse elimination, Knudsen, 2004). While the assumptions about learning rates help to explain the behavioural data in this study, they also show how structural priming may be related to neural mechanisms that support changes in plasticity over development.

\section{Conclusion}

Exploring the effect of verb-syntactic preferences on priming in three age groups has allowed us to assess when children start to develop knowledge of verb argument structure, and to test two possible learning mechanisms that might be involved in the development of verb-structure links. Although the lexical boost was apparent only in adults, the identity of the target verb (target verb bias) and the prime verb (prime surprisal) influenced both children's and adults' structure choice from the earliest age tested. Thus, our results suggest that children acquire abstract knowledge of structure and develop knowledge about verb-argument structure preferences early in acquisition. Children's performance, however, was not adultlike; the effect sizes for target verb bias increased, and for prime surprisal decreased, over development. Taken as a whole, the pattern of development leads us to propose that the process of verb argument structure learning is best explained by a mechanism that uses error-based learning with a variable learning rate.

\section{Acknowledgments}

Michelle Peter, Franklin Chang, Julian Pine, and Caroline Rowland are members of the International Centre for Language and Communicative Development (LuCiD) at the University of Liverpool. The support of the Economic and Social Research Council [ES/L008955/1] is gratefully acknowledged. Financial support for this research was supported by a Faculty-funded doctoral studentship awarded to Michelle Peter by the University of Liverpool. We wish to thank the schools and children who took part in this study, and to the students who helped to collect the data. Thanks also go to the audiences at the AMLaP conference (Riva del Garda, Italy) in September, 2012 and at BUCLD in November, 2012 for their helpful comments on an earlier version of this paper. For information on how to access the materials for this research, please contact the first (corresponding) author.

\section{Appendix A}

\section{Experimental items}

Prime sentences before the slash are presented in the DOD structure; those after the slash are in the PD structure. The four prime verbs are given in parentheses. Target stems used the same combination of agent, verb, recipient, and theme as prime sentences (e.g., the target stem, "Piglet GAVE..." was designed to elicit either Piglet gave Tigger a puppy or Piglet gave a puppy to Tigger).

1. The king (GAVE/ SHOWED/ BROUGHT/ SENT) the queen a baby/a baby to the queen.

2. The boy (GAVE/ SHOWED/ BROUGHT/ SENT) the girl a fish/ a fish to the girl.

3. Wendy (GAVE/ SHOWED/ BROUGHT/ SENT) Bob a puppy/a puppy to Bob.

4. Dora (GAVE/ SHOWED/ BROUGHT/ SENT) Boots a rab$\mathrm{bit} / \mathrm{a}$ rabbit to Boots.

5. The prince (GAVE/ SHOWED/ BROUGHT/ SENT) the princess a baby/a baby to the princess.

6. The king (GAVE/ SHOWED/ BROUGHT/ SENT) the queen a cat/a cat to the queen.

7. Piglet (GAVE/ SHOWED/ BROUGHT/ SENT) Tigger a puppy/a puppy to Tigger.

8. Wendy (GAVE/ SHOWED/ BROUGHT/ SENT) Bob a rabbit/ a rabbit to Bob.

Filler items
1. Boots was flying

2. The princess jumped

3. Piglet and Tigger bounced

4. The king and queen waved

5. Tigger was washing

6 . The prince was rocking

7. Piglet waved

8. The cat was swinging

9. Dora was flying

10. Bob was swinging

11. The princess and the cat were rocking

12. Dora and Boots waved

13. Bob was flying

14. The prince jumped
17. The king and queen bounced

18. Piglet jumped

19 . Wendy was flying

20. Dora was washing

21. The boy waved

22. Boots pointed at Dora

23. Wendy and Bob jumped

24. Dora was swinging

25 . The girl waved

26. Wendy pointed at Bob

27. Boots was washing

28. Piglet was rocking

29. The cat bounced

30. Bob jumped 


\section{Tigger was rocking \\ 16. The queen waved \\ 31. Boots waved at Dora and the baby 32. The king pointed at the queen}

\section{References}

Alishahi, A., \& Stevenson, S. (2008). A computational model of early argument structure acquisition. Cognitive Science, 32, 789-834.

Baayen, R. H., Davidson, D. J., \& Bates, D. M. (2008). Mixed-effects modelling with crossed random effects for subjects and items. Journal of Memory and Language, 59, 390-412.

Barr, D. J., Levy, R., Scheepers, C., \& Tily, H. J. (2013). Random effects structure for confirmatory hypothesis testing: Keep it maximal. Journal of Memory and Language, 68, 255-278.

Bencini, G. M. L., \& Valian, V. V. (2008). Abstract sentence representations in 3-year olds: Evidence from language production and comprehension. Journal of Memory and Language, 59, 97-113.

Bernolet, S., \& Hartsuiker, R. J. (2010). Does verb bias modulate syntactic priming? Cognition, 114, 455-461.

Bliss, T. V. P., \& Collingridge, G. L. (1993). A synaptic model of memory: Long term potentiation in the hippocampus. Nature, 361, 31-39.

Bock, K. (1986). Syntactic persistence in language production. Cognitive Psychology, 18, 355-387.

Bock, K. (1989). Closed-class immanence in sentence production. Cognition, 31, 163-186.

Bock, K., \& Griffin, Z. M. (2000). The persistence of structural priming: Transient activation or implicit learning? Journal of Experimental Psychology: General, 129, 177-192.

Campbell, A., \& Tomasello, M. (2001). The acquisition of English dative constructions. Applied Psycholinguistics, 22, 253-267.

Chang, F., Dell, G. S., \& Bock, K. (2006). Becoming syntactic. Psychological Review, 113, 234-272.

Chang, F., Janciauskas, M., \& Fitz, H. (2012). Language adaptation and learning: Getting explicit about implicit learning. Language and Linguistics Compass, 6, 259-278.

Cleland, A. A., \& Pickering, M. J. (2006). Do writing and speaking employ the same syntactic representations? Journal of Memory and Language, 54, 185-198.

Cohen, J. (1960). A coefficient of agreement for nominal scales. Educational and Psychological Measurement, 20, 37-46.

Coyle, J. M., \& Kaschak, M. P. (2008). Patterns of experience with verbs affect long-term cumulative structural priming. Psychonomic Bulletin E' Review, 15, 867-970.

Crawford, M. L., Harwerth, R. S., Smith, E. L., \& von Noorden, G. K. (1996) Loss of stereopsis in monkeys following prismatic binocular dissociation during infancy. Behavioural Brain Research, 79, 207-218.

Daw, N. W. (1994). Mechanisms of plasticity in the visual cortex. Investigative Opthalmology and Visual Science, 35, 4168-4179.

Dunlap, W. P., Cortina, J. M., Vaslow, J. B., \& Burke, M. J. (1996). Metaanalysis of experiments with matched groups or repeated measures designs. Psychological Methods, 1, 170.

Ellis, A. W., \& Lambon Ralph, M. A. (2000). Age of acquisition effects in adult lexical processing reflect loss of plasticity in maturing systems: Insight from connectionist networks. Journal of Experimental Psychology: Learning, Memory, and Cognition, 26, 1103-1123.

Fine, A. B., \& Jaeger, T. F. (2013). Evidence for implicit learning in syntactic comprehension. Cognitive Science, 37, 578-591.

Gries, S. Th. (2005). Syntactic priming: A corpus-based approach. Journal of Psycholinguistic Research, 34, 365-399.

Gries, S. Th., \& Stefanowitsch, A. (2004). Extending collostructional analysis: A corpus-based perspective on 'alternations'. Internationa Journal of Corpus Linguistics, 9, 97-129.

Gropen, J., Pinker, S., Hollander, M., Goldberg, R., \& Wilson, R. (1989). The learnability and acquisition of the dative alternation in English. Language, 65, 203-257.

Hebb, D. O. (1949). The organization of behaviour: A neuropsychological theory. New York: Wiley.

Hohenstein, S., \& Kliegl, R. (2013). Remef (REMove effects) (version v0.6.10). <http://read.psych.uni-potsdam.de/joomla/attachments/article/12/ remef.v0.6.10.R>.

Jaeger, T. F. (2008). Categorical data analysis: Away from ANOVAs (transformation or not) and towards logit mixed models. Journal of Memory and Language, 59, 434-446.
Jaeger, T. F., \& Snider, N. (2013). Alignment as a consequence of expectation adaptation: Syntactic priming is affected by the prime's prediction error given both prior and recent experience. Cognition, $127,57-83$

Johnson, J. S., \& Newport, E. (1989). Critical period effects in second language learning: The influence of maturational state on the acquisition of English as a second language. Cognitive Psychology, 21, 60-99.

Katz, L. C., \& Shatz, C. J. (1996). Synaptic activity and the construction of cortical circuits. Science, 274, 1133-1138.

Knudsen, E. I. (2004). Sensitive periods in the development of the brain and behavior. Journal of Cognitive Neuroscience, 16, 1412-1425.

Kral, A., \& Sharma, A. (2012). Developmental neuroplasticity after cochlear implantation. Trends in Neuroscience, 35, 111-122.

Landis, J. R., \& Koch, G. G. (1977). The measurement of observer agreement for categorical data. Biometrics, 33, 159-174.

Lenneberg, E. H. (1967). Biological foundations of language. New York: Wiley.

Li, P., Farkas, I., \& MacWhinney, B. (2004). Early lexical development in a self-organizing neural network. Neural Networks, 17, 1345-1362.

MacWhinney, B. (2000). The CHILDES project: Tools for analyzing talk (3rd ed.). Mahwah, NJ: Lawrence Erlbaum Associates.

Mayberry, R. I. (2007). When timing is everything: Age of first-language acquisition effects on second-language learning. Applied Psycholinguistics, 28, 537-549.

McClelland, J. L., McNaughton, B. L., \& O’Reilly, R. C. (1995). Why there are complimentary learning systems in the hippocampus and neocortex: Insights from the successes and failures of connectionist models of learning and memory. Psychological Review, 102, 419-457.

McMurray, B., Horst, J. S., \& Samuelson, L. K. (2012). Word learning as the interaction of online referent selection and slow associative learning Psychological Review, 119, 831-877.

Messenger, K., Branigan, H. P., \& McLean, J. F. (2011). Evidence for (shared) abstract structure underlying children's short and full passives. Cognition, 121, 268-274.

Messenger, K., Branigan, H. P., McLean, J. F., \& Sorace, A. (2012). Is young children's passive syntax semantically constrained? Evidence from syntactic priming. Journal of Memory and Language, 66, 568-586.

Munakata, Y., \& Pfaffly, J. (2004). Hebbian learning and development. Developmental Science, 7, 141-148.

Naito, M. (1990). Repetition priming in children and adults: Age-related differences between implicit and explicit memory. Journal of Experimental Child Psychology, 50, 462-484.

Neter, J., Wasserman, W., \& Kutner, M. H. (1985). Applied linear statistical models: Regression, analysis of variance, and experimental designs. Homewood, IL: Irwin.

Noppeney, U., \& Price, C. J. (2004). An fMRI study of syntactic adaption. Journal of Cognitive Neuroscience, 16, 702-713.

Perfors, A., Tenenbaum, J. B., \& Wonnacott, E. (2010). Variability, negative evidence, and the acquisition of verb argument constructions. Journal of Child Language, 37, 607-642.

Pickering, M. J., \& Branigan, H. P. (1998). The representation of verbs: Evidence from syntactic priming in language production. Journal of Memory and Language, 39, 633-651.

$\mathrm{R}$ Core Team (2012). R: A language and environment for statistical computing. 3-900051-07-0. Vienna, Austria: R Foundation for Statistical Computing. <http://www.R-project.org/>.

Reitter, D., Keller, F., \& Moore, J. D. (2011). A computational cognitive model of syntactic priming. Cognitive Science, 35, 587-637.

Rowland, C. F., Chang, F., Ambridge, B., Pine, J. M., \& Lieven, E. V. M. (2012). The development of abstract syntax: Evidence from structural priming and the lexical boost. Cognition, 125, 49-63.

Rumelhart, D. E., Hinton, G. E., \& Williams, R. J. (1986). Learning internal representations by error propagation. In D. E. Rumelhart \& J. L. McClelland (Eds.). Parallel distributed processing. Explorations in the microstructure of cognition (Vol. 1, pp. 318-362). Cambridge: MIT Press.

Samuelson, L. K. (2002). Statistical regularities in vocabulary guide language acquisition in connectionist models and 15-20-montholds. Developmental Psychology, 38, 1016-1037.

Savage, C., Lieven, E., Theakston, A., \& Tomasello, M. (2003). Testing the abstractness of children's linguistic representations: Lexical and structural priming of syntactic structures in young children. Developmental Science, 6, 557-567.

Shimpi, P. M., Gámez, P. B., Huttenlocher, J., \& Vasilyeva, M. (2007). Syntactic priming in 3- and 4-year-old children: Evidence for abstract representations of transitive and dative forms. Developmental Psychology, 43, 1334-1346. 
Sprondel, V., Kipp, K. H., \& Mecklinger, A. (2011). Developmental changes in item and source memory: Evidence from an ERP recognition memory study with children, adolescents, and adults. Child Development, 82, 1638-1953.

Thothathiri, M., \& Snedeker, J. (2008a). Syntactic priming during language comprehension in three- and four-year-old children. Journal of Memory and Language, 58, 188-213.

Thothathiri, M., \& Snedeker, J. (2008b). Give and take: Syntactic priming during spoken language comprehension. Cognition, 108, 51-68.

Tooley, K. M., \& Bock, K. (2014). On the parity of structural persistence in language production and comprehension. Cognition, 132, 101-136.
Twomey, K., Chang, F., \& Ambridge, B. (2014). Do as I say, not as I do: A lexical distributional account of English locative verb class acquisition. Cognitive Psychology, 73, 41-71.

Wendorf, C. A. (2004). Primer on multiple regression coding: Common forms and the additional case of repeated contrasts. Understanding Statistics, 3, 47-57.

Wiesel, T. N., \& Hubel, D. H. (1965). Extent of recovery from the effects of visual deprivation in kittens. Journal of Neurophysiology, 28, 1060-1072. 